\title{
IMPACTO DEL COMERCIO INFORMAL EN LA ESTRUCTURA ECONÓMICA DE HONDURAS
}

\author{
(1) Nelson Roberto Raudales García, \\ Universidad Nacional Autónoma de Honduras (UNAH) \\ Facultad de Ciencias Económicas, Administrativas y Contables (FCEAC) \\ Instituto de Investigaciones Económicas y Sociales (IIES) \\ Ciudad universitaria, edificio C2, primer piso, Tel/fax: + (504) 2216-6100 Ext. 100894 \\ ORCID 0000-0003-3766-8808 \\ nelson.raudales@unah.edu.hn \\ Rosalina Alvarenga \\ ralvarengaflores@yahoo.com
}

DOI http://dx.doi.org/10.5377/eya.v8i2.5619

\section{RESUMEN}

El presente artículo tiene como finalidad, analizar la contribución del sector informal en la economía del país. Los resultados reflejan que tiene mejor participación la mujer en estas actividades en comparación con los hombres $57 \%$ y $43 \%$ respectivamente. Las principales conclusiones de esta investigación apuntan al apoyo técnico por parte del sector gubernamental y/o de las organizaciones encargadas de contribuir con acciones de emprendimiento a las personas dedicadas a actividades económicas, ya que dicha población opera sin procedimientos administrativos financieros, técnico-operativos. Asimismo, brindar desde los sectores que corresponde el apoyo mediante la aplicación de políticas públicas por ser dicho sector un bastión importante para la economía del país.

Palabras clave: Economía informal, sector económico, emprendimiento 


\title{
IMPACT OF INFORMAL TRADE ON THE ECONOMIC STRUCTURE OF HONDURAS
}

\author{
(1) Nelson Roberto Raudales García, \\ Universidad Nacional Autónoma de Honduras (UNAH) \\ Facultad de Ciencias Económicas, Administrativas y Contables (FCEAC) \\ Instituto de Investigaciones Económicas y Sociales (IIES) \\ Ciudad universitaria, edificio C2, primer piso, Tel/fax: + (504) 2216-6100 Ext. 100894 \\ ORCID 0000-0003-3766-8808 \\ nelson.raudales@unah.edu.hn \\ Rosalina Alvarenga \\ ralvarengaflores@yahoo.com
}

\begin{abstract}
The research follows the objective to analyze the contribution of the informal sector of the economy in the country. The results show than at gender level there is more participation of the women in activities in comparation with the men $57 \%$ and $43 \%$ respectively. The main conclusions of this research is than must be more technical support of the goverments sector or the non-government organizations responsible of contributing with entrpreneurship actions for persons dedicated to economical activities due to than this people work without administrative procedures financials, technical. Operational inefficient, also to give since the sectors than compete support it is up to the aplications of publics political for sector and be a bulwark for the economy of the country.
\end{abstract}

Keywords: Informal Economy, economical sector, entrpreneurship

1 Corresponding author

142 Revista Economía y Administración (E\&A) / VoL. 8 


\section{INTRODUCCIÓN}

El propósito del presente trabajo es describir qué factores que inciden directa e indirectamente en el desarrollo del comercio informal y, si los actores involucrados en dicha actividad económica cuentan con apoyo (técnico, financiero, entre otros) de instituciones para desarrollarse. El abordaje procurará demostrar o caracterizar cual es la situación actual del desarrollo de oportunidades en el mercado laboral que se vislumbra en el Distrito Central, Francisco Morazán.

La economía informal es considerada como una alternativa para obtener ingresos por parte de los núcleos o estructuras familiares de familias con recursos bajos o ingresos medios, sin ningún beneficio social a cambio, sobresaliendo para la población más pobre y con menos posibilidades de sobrevivencia en su vida a largo plazo.

Una alternativa de generación de mejores ingresos familiares y/o una forma de compensar un status de desempleo, entre otros fines, lo que le da una connotación específica en el ámbito nacional; Otro factor que interviene y lo impulsa es el exceso de regulaciones para iniciar un negocio, que implica un proceso retardado, costoso e inaccesible para la población con menos recursos; además de la falta de apoyo de créditos para micro, pequeñas y medianas empresas, obstáculos para creación de puestos de trabajo formales, y crea una forma de exclusión de un segmento importante de la población.

\section{I.- MARCO CONCEPTUAL}

El concepto de economía informal surgió en el tercer mundo, como resultado de una serie de estudios sobre el mercado laboral urbano en África (Keith Hart, 1973); el antropólogo económico que acuñó el término, la consideró como una forma de expresar "La brecha entre mi experiencia en esa región y todo lo que había aprendido hasta ese momento en Inglaterra".

Según el autor, era evidente que las observaciones empíricas sobre el espíritu empresarial popular en
Accra9 y otras capitales africanas no tenían nada en común con los conocimientos adquiridos a través del "discurso occidental sobre el desarrollo económico, cuando el ser humano comienza a preocuparse acerca del modo como se ha adquirido un conocimiento, o cuando intenta encontrar un conocimiento nuevo, se presentan interrogantes.

Refiriéndose al tema que ocupa la presente investigación, el comercio informal en Tegucigalpa $\mathrm{y}$ sus consecuencias en el mercado laboral se puede mencionar la teoría administrativa causal, Humanista, situacional de contingencia (Taylor, F. 1911).

Siguiendo esta teoría que se refiere a diferentes modelos organizacionales, dependiendo de factores externos; y aspectos que no se tienen control sobre ellos, debido a la práctica que realizan en el comercio informal, sin la aplicación de estrategias comerciales estructuradas.

Según algunos autores de esta teoría proponen que no hay nada absoluto en las organizaciones ni en la teoría administrativa en todo lo relativo mencionado y depende de un factor determinante. "Alfred Chander, Fred E. Fielder, Robert Houss, Paul R. Lawrence, Jay W Lorch, G.M, Stalker, Tombur, principales representantes de la teoría situacional de contingencia". Sin embargo, parece contradicción señalar que ninguna teoría administrativa es única para alcanzar los objetivos esperados en una organización, se refiere a que todos los tipos de decisiones dependen del entorno donde se desarrolla la misma.

(Skinner, 1970) menciona en sus escritos sobre la teoría situacional que:" La conducta opera sobre el ambiente externo para provocar algún cambio en el ambiente, entonces el cambio ambiental será contingente en relación con aquella conducta".

Siguiendo este pensamiento del autor, en comercio informal se observa que la mencionada teoría "situacional de contingencias" en las ventas ambulatorias situadas en diferentes lugares y sitios de Tegucigalpa Distro Central convirtiéndose así estos comerciantes en líderes familiares por su

\footnotetext{
9 Acara es la mayor ciudad de Ghana y su capital desde 1877.
}

Revista Economía y Administración (E\&A) / VOL. 8143 
lucha contra las necesidades de empleo existentes en Honduras. En base a los autores antes mencionados las relaciones humanas se practican en comercio informal es abierta total al ambiente, la cual se desarrolla sin ninguna estructura definida poniendo en práctica el uso de diversidad de variables ambientales incontrolables, (cultura, demografía, ecología) competitivas entre sí y a la exposición de entidades que regulan el comercio informal en la ciudad de Tegucigalpa y otros desafíos externos sin ninguna contemplación.

La operación del sistema informal es totalmente rutinaria y de improvisación, pues lo que estos comerciantes buscan es el sostén diario para sobrevivir, utilizando el efectivo del momento como únicos ingresos. Sin embargo, Mario Bunge $^{10}$ se dirige a encontrar las diferentes teorías que se pude utilizar para determinar los diferentes tipos, Bunge tiende a dar mayor importancia al tema científico, según su escrito es allí donde se encontrará la verdad de teorías con respecto al tema por investigar"

En su informe la Oficina Internacional del Trabajo (OIT), (Hart, 1973) postulado un modelo dual de oportunidades de ingresos para la fuerza de trabajo urbana, basado principalmente en la distinción entre el empleo remunerado y el trabajo por cuenta propia.

El concepto de "informalidad" se aplicó a este último tipo de actividad. Este autor señaló la notable dinámica y diversidad de estas actividades que para él "Iban mucho más allá de los limpiabotas y los vendedores de fósforos". Posteriormente, esta caracterización dinámica del sector informal terminó desdibujándose a medida que el concepto se fue institucionalizando en la burocracia de la OIT, que redefinió la informalidad y la convirtió en sinónimo de pobreza.

Se consideró que la economía informal era una "modalidad urbana" caracterizada por:

1) La exigüidad de los obstáculos al ingreso, en lo que La economía informal se refiere a las aptitudes, el capital y la organización; 2) la propiedad familiar de las empresas; 3) lo reducido de la escala de operaciones; 4) el empleo de métodos de producción de gran densidad de mano de obra y de tecnologías anticuadas; y 5) la existencia de mercados no regulados y competitivos. (Rosenbluth, G. 1994).

De esta definición se derivan otras características, como los bajos niveles de productividad y la poca capacidad de acumulación (Tokrnan, G. 1984). En publicaciones posteriores del Programa Regional del Empleo para América Latina y el Caribe (PREALC) de la Organización Internacional del Trabajo (OIT),(Regional Employment Program for Latin America and the Caribbean, 1982) el empleo en el sector informal se denominó sistemáticamente "subempleo" y se supuso que quienes participaban en él no lograban ingresar a la economía moderna (Tokman, 1984).

Esta caracterización de la economía informal, como segmento excluido de las economías menos desarrolladas" ha sido consagrada en muchos estudios sobre la pobreza urbana y los mercados de trabajo realizados por la OIT, el PREALC y el Banco Mundial.

La caracterización negativa del sector informal ha sido cuestionada por otros estudiosos del tema, que opinan todo lo contrario. Desde este punto de vista alternativo, las actividades informales se consideran un signo de dinamismo empresario popular, descrito por Hart "como una instancia en que la gente retoma en sus propias manos parte del poder económico que trataron de negarle los agentes centralizados".

El economista peruano Hernando de Soto reformuló el concepto original de Hart y le dio nuevo impulso. En su libro titulado The Other Path (1987), Soto define la informalidad como la respuesta popular a la rigidez de los Estados "mercantilistas" predominantes en Perú y otros países de América Latina, que sobreviven otorgando el privilegio de participar legalmente en la economía a una pequeña élite. 
Así, a diferencia de la concepción sostenida por la OIT y el PREALC, que la consideran un mecanismo de supervivencia en respuesta a la falta de creación de empleos suficientes en la economía moderna, las empresas informales representan la irrupción de fuerzas reales del mercado en una economía aprisionada por las regulaciones del Estado.

\section{I.1.- Definiciones contemporáneas}

El fuerte componente normativo vinculado con estos análisis contrapuestos del sector informal en el tercer mundo no está del todo ausente en los correspondientes a los países industrializados, pero allí las investigaciones permiten lograr una definición más precisa y menos tendenciosa. Parece existir un consenso cada vez mayor entre los investigadores del mundo desarrollado que el campo de aplicación del término sector informal abarca "las acciones de los agentes económicos que no adhieren a las normas institucionales establecidas o a los que se niega su protección". O bien, incluye "todas las actividades generadoras de ingresos no reguladas por el Estado en entornos sociales en que están reguladas actividades similares".

En estas definiciones "No se emite un juicio de valor a priori sobre estas actividades" dejando que la cuestión se resuelva a través de las investigaciones empíricas. En tal sentido, parecen ser superiores, desde un punto de vista heurístico, a las usadas en el mundo en desarrollo, en las que se anuncia desde un principio las conclusiones a las que debe llegarse.

No obstante, aún las definiciones neutras tienen una limitación. Son insuficientes para abarcar la enorme amplitud de la temática a la que hacen referencia. Aplicando el enfoque de la nueva economía institucional, según (Feige, 1990) propone una taxonomía que permite especificar con mayor precisión el universo que se está considerando. (Portes, A. 2004).

Esta clasificación se realiza teniendo en cuenta las normas institucionales de las que se hace caso omiso en una actividad económica determinada.
Con el término genérico economía subterránea se distingue cuatro sub formas: CEPAL - SERIE Políticas sociales $\mathrm{N}^{\mathrm{o}} 100$.

1) La economía ilegal, que abarca la producción y distribución de bienes $\mathrm{y}$ servicios prohibidos por la ley. Comprende las actividades como el narcotráfico, la prostitución y los juegos de azar ilegales.

2) La economía no declarada que consiste en la realización de acciones que "soslayan o evaden las normas impositivas establecidas en los códigos tributarios". El monto de los ingresos que deberían declararse a las autoridades impositivas, pero no se declaran, representa un ejemplo sumario de este tipo de economía subterránea.

3) La economía no registrada, que comprende las actividades que transgreden los requisitos de los organismos estadísticos del Estado en materia de declaración. Su medida estricta es el monto de los ingresos que deberían registrarse en los sistemas de cuentas nacionales, pero no se registra.

La economía informal, que abarca las actividades económicas que hacen caso omiso del costo que supone el cumplimiento de las leyes y las normas administrativas que rigen las "relaciones de propiedad, el otorgamiento de licencias comerciales, los contratos de trabajo, los daños, el crédito financiero y los sistemas de seguridad social" y están excluidas de la protección de aquellas. (Portes, A. (2010)

Evidentemente, existe bastante superposición entre estas diversas formas ya que la mayoría de las actividades consideradas informales tampoco se registran o declaran. La distinción conceptual más importante se produce entre las actividades informales y las ilegales, pues cada una de ellas posee características distintivas que las diferencian entre sí.

Los sociólogos generalmente reconocen que lo legal y lo delictivo, lo mismo que lo normal o lo anormal, son categorías definidas socialmente, y que pueden cambiar. No obstante, las actividades 
ilícitas suponen la producción y comercialización de bienes, definidos en un lugar y un momento determinados como ilegales, mientras que en la mayoría de los casos las actividades informales están relacionadas con bienes lícitos.

La diferencia fundamental entre la economía formal y la informal no guardan relación con las características del producto final sino con la forma en que éste es producido o intercambiado. Así, las prendas de vestir, los alimentos que se sirven en un restaurante o los circuitos integrados de las computadoras, todos productos totalmente lícitos pueden originarse en sistemas productivos regulados por la ley o en entornos en los que se hace caso omiso de las normas oficiales.

Al distinguir explícitamente estas tres categorías (actividades formales, informales e ilegales) se puede explorar sistemáticamente las relaciones existentes entre ellas, una tarea difícil cuando se confunde lo informal con lo ilegal. Por ejemplo, (Hart, K. ,1973). analizó los efectos profundos de la economía de la droga liviana sobre los sectores formal e informal de ese país. Además estudió interrelaciones similares en la ex Unión Soviética y sus países satélites de Europa oriental.

\section{II.- RESULTADOS}

Los objetivos iniciales planteados para el diseño de esta investigación fueron enriquecidos y ampliados ante la falta de información apropiada para este sector productivo de nuestra economía que podría tener un gran potencial si el gobierno y el sector empresarial le brindara apoyo requerido especialmente en lo relacionado con la asistencia técnica en la comercialización de sus productos y financiamiento e implementos tecnológicos.

Tal como se mencionó previamente en el resumen de la investigación se ha podido observar que las personas dedicadas al comercio informal es población con edades sumamente jóvenes, lo que les impide, dificulta y limita el desarrollo del capital humano por parte de esta población activa dedicada al comercio de esta naturaleza (más detalle puede reflejarse en tabla No. 1)

Tabla No. 1 - Tegucigalpa población según campo de edades dedicada al comercio informal, año 2016:

\begin{tabular}{|l|r|r|}
\hline \multicolumn{1}{|c|}{ Rango de edad } & \multicolumn{1}{|c|}{ Frecuencia } & \multicolumn{1}{c|}{ Porcentaje } \\
\hline 15- 20 años & 34 & 8.9 \\
\hline 21-30 años & 103 & 26.9 \\
\hline $31-50$ años & 178 & 46.5 \\
\hline $\begin{array}{l}51 \text { años en } \\
\text { adelante }\end{array}$ & 68 & 17.8 \\
\hline Total & 383 & 100.0 \\
\hline
\end{tabular}

Fuente: Elaboración propia en base a Encuesta aplicada a personas activas en el comercio informal en el Distrito Central, Francisco Morazán, 2016.

Los datos mostrados en la tabla anterior (No. 1), muestran que la población involucrada en actividades dedicadas al comercio de bienes y servicios o lo que se denomina "comercio informal", es una población con características sumamente jóvenes donde uno mueve de cada diez personas dedicadas a dicho rubro es población que se encuentra en edades comprendidas entre los 15-20 años de edad, estas personas por su grupo etario deberían estar dedicándose al estudio, por lo tanto se les está limitando el desarrollo de las facultades y capacidades o la aprehensión de conocimientos que les permita tener la posibilidad de insertarse en actividades económicas del sector formal, disfrutando principalmente de prebendas o beneficios sociales.

Adicionalmente, se puede observar que existe también una de cada cuatro personas dedicadas también al sector informal en edades entre

146 Revista Economía y Administración (E\&A) / VOL. 8 
los 21-30 años lo que representa un $26 \%$ aproximadamente, lo cual posiblemente sea población que ya dispone de responsabilidades económico-familiares de sustento alimenticio para el núcleo de personas con el cual conviven.

En cuanto a los datos de resultados de género son más las mujeres las que se dedican a las actividades orientadas al comercio informal; $56 \%$ aproximadamente se encuentran en actividades de comercialización de bienes y servicios lo que representa un 57\% aproximadamente. Mientras que en el caso de los hombres se insertan en esta actividad informal un $43 \%$ aproximadamente (más detalle ver tabla No. 2).

Tabla No. 2. - Tegucigalpa, Sexo de las personas dedicada al comercio informal, año 2016

\begin{tabular}{|l|r|r|}
\hline \multicolumn{1}{|c|}{ Sexo } & \multicolumn{1}{c|}{ Frecuencia } & \multicolumn{1}{c|}{ Porcentaje } \\
\hline Femenino & 216 & 56.4 \\
\hline Masculino & 167 & 43.6 \\
\hline Total & 383 & 100.0 \\
\hline
\end{tabular}

Fuente: Elaboración propia en base a Encuesta aplicada a personas activas en el comercio informal en el Distrito Central, Francisco Morazán, 2016.

Se vuelve pertinente observar tal como se planteó previamente, la ubicación de los negocios o los comercios dedicados al comercio informal en el Distrito Central, departamento de Francisco Morazán. El principal punto geográfico o mercado capitalino en donde las personas se dedican al desarrollo de sus actividades de bienes y servicios es el mercado Zonal Belén, en este uno de cada tres personas dedicadas al comercio informal se encuentran en dicho mercado, lo que representa un $33 \%$ aproximadamente y, convirtiendo a este mercado como la principal y la primera plaza donde se desarrolla el comercio informal.

Los datos relacionados a las ubicaciones física o geográfica en donde se encuentran las personas que desarrollan su actividad informal se ubican principalmente en el Distrito Central que la ubican en segunda posición y en donde tres de cada diez de estos negocios de bienes y servicios se ubican muy cercanos a la ciudad céntrica de Tegucigalpa, tal proporción representa aproximadamente (más detalle se puede observar en la tabla No. 3). Llama la consideración que la tercera ciudad en mostrar altos índices de concentración de personas dedicadas al comercio informal es la ciudad o la Colonia Kennedy, donde diez de cada cien personas se dedican al rubro del comercio informal de bienes y servicios lo que representa un $10 \%$ aproximadamente. Las demás ciudades del Distrito Central muestran porcentajes bajos, sin embargo, son de consideración también en virtud de que en dichas comunas la población tiene el derecho de poder ganarse el sustento económico y muy bien dedicándose a actividades productivas remuneradas, lo cual contribuye al mantenimiento económico del país, así como al sustento de las familias. 
Tabla No. 3. - Tegucigalpa, ubicación de las personas dedicada al comercio informal, año 2016

\begin{tabular}{|l|r|r|}
\hline $\begin{array}{c}\text { Comunidades de } \\
\text { operación del comercio } \\
\text { informal }\end{array}$ & Frecuencia & Porcentaje \\
\hline La travesía & 4 & 1.0 \\
\hline Col. San Miguel & 1 & .3 \\
\hline El centro de la ciudad & 107 & 27.9 \\
\hline El Guanacaste & 19 & 5.0 \\
\hline Zonal Belén & 124 & 32.4 \\
\hline Alrrededores del mercado & 1 & .3 \\
\hline Kennedy & 38 & 9.9 \\
\hline Bulevar Suyapa & 28 & 7.3 \\
\hline Centro América & 1 & .3 \\
\hline Feria del agricultor & 19 & 5.0 \\
\hline Col. Villa Nueva & 11 & 2.9 \\
\hline villas del sol & 2 & .5 \\
\hline Mercado Jacaleapa & 6 & 1.6 \\
\hline El prado & 3 & .8 \\
\hline Col. Alameda & 1 & .3 \\
\hline El manchen & 12 & 3.1 \\
\hline El Picacho & 1 & .3 \\
\hline Bulevar Juan Pablo & 5 & 1.3 \\
\hline Total & 383 & 100.0 \\
\hline
\end{tabular}

Fuente: Elaboración propia en base a Encuesta aplicada a personas activas en el comercio informal en el Distrito Central, Francisco Morazán, 2016.

Al observar los principales rubros económicos de comercialización de bienes y servicios a los que se dedica la población que desarrolla actividades comerciales se encuentra que: tres de cada cinco negocios se dedican a la comercialización de productos alimenticios lo que representa un $60 \%$ aproximadamente. El segundo rubro comercial de las personas dedicadas al comercio informal se encuentra la venta de productos varios.

De igual manera, se ubican en tercera posición aquellos negocios dedicados a la comercialización de venta y distribución de accesorios donde un $10 \%$ de dichos negocios se dedican a la actividad mencionada. (Más detalle ver tabla No. 4) 
ISSN 2219-6722 / ISSNE 2222-2707

Tabla No. 4. - Tegucigalpa, rubros comerciales de las personas dedicada al comercio informal, año 2016:

\begin{tabular}{|l|r|r|}
\hline \multicolumn{1}{|c|}{ Rubro comercial } & Frecuencia & Porcentaje \\
\hline Alimentos & 219 & 57.2 \\
\hline Ropa & 28 & 7.3 \\
\hline Calzado & 19 & 5.0 \\
\hline Abarrotería & 10 & 2.6 \\
\hline Accesorios & 41 & 10.7 \\
\hline Chicles y periódico & 4 & 1.0 \\
\hline Plásticos & 10 & 2.6 \\
\hline Venta de plantas & 4 & 1.0 \\
\hline Trabajos de madera & 1 & .3 \\
\hline Ventas varias & 47 & 12.3 \\
\hline Total & 383 & 100.0 \\
\hline
\end{tabular}

Fuente: Elaboración propia en base a Encuesta aplicada a personas activas en el comercio informal en el Distrito Central, Francisco Morazán, 2016.

Cuando se observa la cantidad de empleados que tienen las personas que se dedican al comercio informal se puede reflejar que la mayoría, siete de cada diez personas con este tipo de negocios no tienen ningún empleado a su cargo, lo que aunado a la precariedad laboral determina que estas personas no tienen la capacidad de poder contratar a empleados que les ayuden para el desarrollo de la actividad orientada a la venta de productos y servicios, esto representa un $70 \%$ aproximadamente. (Ver tabla No 5).
Otra característica de la cantidad de personas empleadas en el comercio informal refleja también que apenas quince de cada cien negocios cuentan apenas con un empleado, lo que representa un $14 \%$ aproximadamente. En este sector micro empresarios contratan entre tres a cinco empleados loque representa porcentajes bajos de participación económica o de generación de empleo tal como se comentó, dichas categorías representan entre un 0.3 a $2.3 \%$ del total de negocios que contratan personas que les contribuyan con el apoyo de las actividades de comercio de bienes y servicios.

Tabla No. 5. - Tegucigalpa, número de empleados de las personas dedicada al comercio informal, año 2016.

\begin{tabular}{|l|r|r|}
\hline Cantidad de empleados & Frecuencia & Porcentaje \\
\hline Ninguno & 271 & 70.8 \\
\hline 1 empleado & 55 & 14.4 \\
\hline 2 empleados & 44 & 11.5 \\
\hline 3 empleados & 9 & 2.3 \\
\hline 4 empleados & 3 & .8 \\
\hline 5 empleados & 1 & .3 \\
\hline Total & 383 & 100.0 \\
\hline
\end{tabular}

Fuente: Elaboración propia en base a Encuesta aplicada a personas activas en el comercio informal en el Distrito Central, Francisco Morazán, 2016.

Al continuar analizando los datos relacionados al tipo de propietario o persona que se dedica al desarrollo de las actividades orientadas al comercio informal se refleja que la mayoría, nueve de cada diez personas involucradas en el comercio informal son dueñas de dichos negocios, lo que representa un $95 \%$ aproximadamente, (Ver tabla No. 6). Asimismo, se refleja que de 100 personas son empleados o trabajadores independientes lo que representa apenas un 5\%. 
Tabla No. 6 - Tegucigalpa, tipo de propietario de las microempresas dedicadas al comercio informal, año 2016.

\begin{tabular}{|c|c|c|}
\hline Tipo propietario & Frecuencia & Porcentaje \\
\hline Dueño & 363 & 94.8 \\
\hline Trabajador & 20 & 5.2 \\
\hline Total & 383 & 100.0 \\
\hline
\end{tabular}

Fuente: Elaboración propia en base a Encuesta aplicada a personas activas en el comercio informal en el Distrito Central, Francisco Morazán, 2016.

Al analizar el clima sobre la percepción económica que se presenta en la economía informal, se vislumbra de que dos de cada tres micro empresarios dedicados o trabajando en el sector informal observan que la percepción sobre la economía del país y específicamente de la operación del sector de las micro indican que la situación es mala, lo que representa un $67 \%$ aproximadamente. (Ver tabla No. 7). Otras respuestas de percepción de los micro empresarios reflejan que más de uno de cada cuatro personas activas en el rubro consideran la situación con niveles de inestabilidad lo que representa un $29 \%$ aproximadamente

Tabla No. 7- Tegucigalpa, percepción de los microempresarios dedicadas al comercio informal, año 2016.

\begin{tabular}{|l|r|r|}
\hline \multicolumn{1}{|c|}{ Percepción } & Frecuencia & Porcentaje \\
\hline Muy mala & 259 & 67.6 \\
\hline Inestable & 110 & 28.7 \\
\hline Buena & 7 & 1.8 \\
\hline Estable & 7 & 1.8 \\
\hline Total & 383 & 100.0 \\
\hline
\end{tabular}

Fuente: Elaboración propia en base a Encuesta aplicada a personas activas en el comercio informal en el Distrito Central, Francisco Morazán, 2016.

\section{DISCUSIÓN}

A partir de los resultados previamente apuntados es plausible determinar que en ausencia de empleo tanto en Tegucigalpa, como en sus otras principales ciudades los detonantes del crecimiento del comercio informal que a diario se presentan en las calles. Esta situación se ha extendido no solo en el Distrito Central, sino en otros centros urbanos como San Pedro Sula y La Ceiba, donde las autoridades municipales se encuentran implementando operativos para sacar de las plazas públicas a los vendedores.

Solo en la capital existen unas 78,000 Mi pyme que se dedican principalmente al comercio y su crecimiento anual es de 20,000 y cada año el mismo número sale del mercado. Los micro empresarios aún continúan en la mesa del debate con el gobierno con el objetivo de reducir el gravamen de este impuesto que reduciría sus ingresos en 0.3 por ciento por cada 120,000 Lempiras.

De acuerdo con Barrientos, M. (2015), representante gubernamental que apoya la Asociación Nacional de Medianos y Pequeños Industriales de Honduras (ANMPIH), estimó que tres de diez empresas sí logran subsistir después de dos años de funcionamiento. De las 60 mil MI PYME solo un $2 \%$ de las pequeñas y medianas empresas logran exportar. "Por eso tenemos ese déficit comercial de más de mil millones de dólares en Centroamérica, porque no hemos aprovechado el tratado de libre comercio".

Barrientos manifestó que "existen convenios con BANADESA los cuales fueron posible por la mediación de la Secretaría de Desarrollo

150 Revista Economía y Administración (E\&A) / VoL. 8 
Económico y Social quienes brindaron asesorías a estos microempresarios, los contenidos de estas capacitaciones se centran en varios temas de interés para los participantes".

Además existen planes de desarrollo empresarial y de mercadeo, para poder acceder a créditos destinados para este sector, para empresas que deseen consolidar deudas o fortalecer sus negocios a tasas bajas de interés. Esto se hace con el propósito de darle la oportunidad a $120 \mathrm{mil}$ empresas que puedan salir de la central de riesgo y muchas veces las políticas de la Comisión de Bancos y

Seguros obstaculizan la política crediticia de BANADESA.

Lo anterior representa que un alto porcentaje de pequeños empresarios no puedan acceder a estos créditos, porque están en la central de riesgo y ninguna entidad crediticia les apoyaría, apuntó, Rodríguez.

\section{CONCLUSIONES}

A continuación se enuncian las principales consideraciones o conclusiones encontradas del trabajo de investigación, las mismas se validaron mediante la generación y análisis de los resultados obtenidos de la propuesta exploratoria realizada.

1. Los hallazgos revelan que sí existe una relación de influencia directa generada por los altos índices de desocupación que impacta en un alza del comercio Informal. En los últimos años se han reportado representativas alzas de este tipo de actividades que subsidian de manera básica la vida de los comerciantes.

2. Las actividades de Comercio Informal, en una porción representativa, albergan un alto porcentaje de personas desocupadas en el Distrito Central.

3. Con una opinión generalizada los comerciantes aseguran estar aportando a solventar un problema que el gobierno no está en capacidad de enfrentar: la reducción de desempleados, reduciendo los índices de pobreza.

4. Los comerciantes que se dedican a actividades informales SÍ están dispuestos a formalizar sus negocios a cambio de lograr beneficios como:
a. Inclusión financiera
b. Fondos para adquisición de viviendas
c. Inscripción a sistemas de salud.

Pese a la falta de participación y de apoyo por los diferentes entes organizaciones sindicales, quienes terminan perdidos en el horizonte anteponiendo intereses propios y personales impiden cualquier proyecto de desarrollo o de mejora para logar un porcentaje de participación de negocios en economía informal, que están en el olvido, pero que son un fuerte motor a la economía de este país en general.

\section{RECOMENDACIONES}

Después de concluida la presente exploración de la investigación, caracterizado por ser un trabajo dinámico, que trata la vez, de una enorme problemática social fuerte que genera el país y se enfrenta con muy pocas opciones de solución, el Comercio Informal atrae los desocupados a buscar una forma alternativa que les permita tener una vida digna.

1. El Gobierno principalmente debe buscar una estrategia de acercamiento con beneficios (Créditos, mejoras de locales, acceso a salud) y obligaciones (paguen al gobierno sus respectivos tributos por sus ingresos) a los comerciantes informales que a través de las diferentes organizaciones sindicales a las que ya pertenecen, mismas que deben desvincularse de intereses políticos y personales, para tener la garantía de una exitosa ejecución de 
estrategias.

2. Las fuentes de empleo que actualmente ofrece el Gobierno no llegan a satisfacer las necesidades básicas de los participantes, no dan estabilidad laboral, por ejemplo: Con chamba vivís mejor, Chamba Joven, por considerarse que los mismos no contribuyen al desarrollo íntegro de los empleados. El gobierno podría, en cambio promover programas integrales atractivos.

3. Crear un ente descentralizado, con la suscripción y participación de todos o en su mayoría de los comerciantes informales donde se les genere opciones de mejorar sus condiciones.

4. A través del ente descentralizado capacitar, educar y crear una cultura de emprendedurismo en los comerciantes, con el apoyo del Gobierno. Una vez incluido financieramente, los pequeños negocios tendrían un porcentaje alto de crecer y convertirse en empresas que empleen un gran número de desempleados.

5. En los últimos días el país ha vivido históricamente despido masivo de personal de instituciones públicas, en su mayoría personas que el mercado laboral tan competitivo no absorberá. Por diferentes razones, los ex empleados buscan la forma de solventar su costo de vida. Con la creación, de una institución o un ente que regule las actividades de comercio informal podría evitarse el crecimiento descontrolado de estas actividades y a través de una asistencia oportuna, reubicar o dar oportunidad a quienes ya de forma legal se dedicarían a hacer actos de comercio al estar suscritos a este ente.

6. La falta de gestión y apoyo por parte del Gobierno a la pequeña y mediana empresa, a largo plazo priorizar las necesidades de los comerciantes puede convertirse en empresas que escalan de tamaño, amplían sus negocios y por ende ocupan más personal para desarrollar las diferentes actividades que requiere la empresa, reduciendo así el número de desempleados y mejorando las condiciones de vida, generando al país millones de tributos y una actividad económica dinámica, ya que generando fuentes de empleo permiten un mayor poder adquisitivo y por ende incremento de consumo local. 


\section{REFERENCIAS}

Barrientos, M. (2015). Desempleo en Honduras: mayo 2015. Recuperado a partir de http://maryori2427.blogspot.com/2015_05_01_ archive.html

Feige, L. (1990). Defining and estimating underground and informal economies: The new institutional economics approach. Elsevier.

Frederick Winslow Taylor

(1911) Principles of Scientific Management.

Hart, K. (1973). Informal income opportunities and urban employment in Ghana. Journal of Modern African Studies 11, 61-89.

PREALC. (1982). Regional Employment Program for Latin America and the Caribbean: Participations laboral: experiencias en Perú y Chile (1a ed). Santiago, Chile: Oficina Internacional del
Trabajo, PREALC.

Portes, A. (2004) La sociología en el continente: convergencias pretéritas y una nueva agenda de alcance medio.

Rosenbluth, G. (1994). Informalidad y pobreza en América Latina [Text]. Recuperado 21 de julio de 2016, a partir de http://www.cepal.org/ es/publicaciones/11941-informalidad-y-pobrezaen-america-latina.

Portes, A. (2010). Economic sociology: a systematic inquiry. Princeton, N.J: Princeton University Press.

Skinner, B. F. (1970). Etimología de enseñanza. En Tecnología de la enseñanza, (pp. 17-23). BCarcelona: Labor.

Tokrnan, G. (1984) Changes in employment and the crisis. 